\title{
Emissive Properties of Materials and its Relation with Roughness
}

\author{
by L. Rozanski* and M. Wieczorowski*
}

\author{
* Poznan University of Technology, Poznan, Poland
}

\begin{abstract}
In the paper analysis of surface topography influence on emissivity of metals was shown. This was performed for infrared band comprising wavelength region of 7.5-13 micrometers. Appropriate characterization and description of object emissivity has a crucial influence on accuracy of IR system for remote temperature measurement, e.g. IR thermography or pyrometry. These properties depend on many factors, including surface topography of material, where especially cavities play a very important role. In references so far emissivity are discussed mainly as influence of type of material (metal, dielectric), its temperature, wavelength or direction of emission. In the paper characterizing emissivity of construction materials in connection with 3D surface topography.
\end{abstract}

\section{Introduction}

The trend in processing and other manufacturing technologies is to utilize more and better sensors for the measurement and control of the processes and material parameter. The measurement and control of the temperatures in industrial processes is important from the standpoints of energy managements, productivity and product quality (total quality, zero-defect manufacturing policy). The radiometric determination of the temperature or utilizing the radiometric phenomena for controlling the process temperature is thus of great practical importance, and is widely used in science and industry [1], [2]. The fact that radiation is a function of obiect surface temperature makes it possible for a remote temperature measurement systems to calculate this temperature. However, the radiation measured by these measuring systems does not only depend on the object temperature but is also a function of its emissivity ( in short, emissivity is a measure of how much radiation is emitted from the object compared to that if it was a perfect blackbody;emissivity is a unitless quantity and spans from 0 to 1 ). Radiation that originates from surroundings is reflected in the object (a radiation thermometer detects the spectral radiance, including both emitted radiance and reflected irradiance, of an opaque object). To measure temperature accuratly, it is therefore necessary to compensate for the effect of a number of different radiation sources. The fallowing object parameters must be supplied: emissivity, ambient temperature, atmospheric temperature, distance and relative humidity of the air. One of the most important parameters is surface emissivity. The radiative properties depend not only on the properties of a material but also on the surface conditions. While data from the literature for the polished metal and/or the entirely oxidized metal can be a useful guide, however, they are not often applicable to practical cases [1]. 


\section{IR thermography}

Temperatures measured in almost all practical industrial applications vary from about $200 \mathrm{~K}$ to about to $3000 \mathrm{~K}$. Objects of such temperatures emit most thermal radiation in infrared (IR) range [1]. Almost all systems used in practice for remote (non-contact) temperature measurement employ the phenomenon of IR radiation that carries information about object temperature. Non-contact thermometers can be divided into pyrometers, line scanners and thermal imagimg devices - IR thermographs ( IR thermal cameras). Pyrometers enable temperature measurement of only a single point; line scanners enable temperature measurement of many points located along a line; IR thermal cameras enable temperature measurement of thousands of points located within a field of view (FOV) of the optical system of the cameras. These cameras offer the greatest capabilities of all mentoned above types of noncontact radiometric thermometers. Modern IR imaging devices enable creation of two-dimensional image of geometrical resolution close to resolution of typical television image with the thermal resolution of the range $0.01{ }^{\circ} \mathrm{C}$. Because they make possible presentation of measurement results in form of electronic image, they are very convenient for users [3]. Therefore IR thermal cameras found numerous applications. These devices are applied in thermal diagnosis where basing surface distribution of temperature it is possible to analyse processes or phenomena. Each process, where the temperature, may be used as the factor of physical process, or temperature may help to measure indirectly other physical values or to be to emergency signal, is the potencial application for IR thermography. The field of the possible application of the IR thermography is very wide and it covers such parts of science and technique as: machine industry, heating, metallurgy, chemical industry, power systems, electronics,... etc [4].

\section{Emissive properties of materials and its relation with roughness}

The value of the measured signals of the IR thermographic systems is a function an object temperature and, among others, is also function an object emissivity [5]. Therefore, to measure the temperature accurately with IR system, it is necessary to know this parameter. Emissivity describes the object's ability to emit thermal radiation; it is expressed as the ratio of the radiation emitted by a object surface to the radiation emited by the blackbody in the same condditions of temperature, direction and spectral band of interest. Normally, object materials and surface treatments exhibibit emissivities ranging from approximately 0.05 to 0.99 . A highly polished surface falls below 0.1 , while an oxidised or painted surface has much higher emissivity.

Generally, emissivity is not a constant, as it depends on several parameters: temperature, viewing angle, wavelenght, contamination or roughness. All totoal radiative properties of materials can only be regarded as fuction of veiwing angle and temperature. Spectral emissivity $\varepsilon(\lambda)$ as a function of wavelenght $\lambda$ decreases for metals, increases for dielectrics and is band-like for gases, liquids and same solids. The chemical and physical changes of the emitting body caused by temperature, time and pressure influence its emissivity. A general characteristic, independent of the kind of material is the variability of emissivity according to surface roughness [6]. Emissivity increases with the increase of roughness. The most popular surface roughness profile parameters are $\mathrm{Ra}$ (avarage roughness parameter) and $\mathrm{Rq}$ (the root mean square roughness). In particular the emmisivity of metal, which is usually low, can considerably increase with roughness. An analysis of the emmssivity as a 
function $R_{q}$ demonstrates that a treatment of the surface in terms of $R_{q}$ is absolutely insufficient to predict radiative properties of materils [7].

\section{Research method}

\subsection{Effective emissivity}

Most of commercially available thermal instruments need the effective emissivity $\varepsilon_{\text {eff }}(\lambda)$ of the tested object as an input parameter [5]. The effective emissivity of the IR system, in spectral band from $\lambda_{1}$ to $\lambda_{2}$, is defined as the mean value of the function of spectrally variable emissivity $\varepsilon(\lambda)$ weighted by the product of function of the spectral object luminance at the temperature $T_{o b} L\left(T_{o b}, \lambda\right)$, the detector relative sensitivity $s(\lambda)$ and the optics transmittance $\tau_{\mathrm{o}}(\lambda)$ :

$$
\varepsilon_{e f}=\frac{\int_{\lambda_{1}}^{\lambda_{2}} \varepsilon(\lambda) s(\lambda) L\left(T_{o b}, \lambda\right) d \lambda}{\int_{\lambda_{1}}^{\lambda_{2}} s(\lambda) L\left(T_{o b}, \lambda\right) d \lambda}
$$

The application of the Eq. (1) for the determination of the effective emissivity improve accuracy of remote temperature measurement with systems of spectrally dependent sensitivity [5]. The value of effective emissivity is required to enter the IR system microcomputer. At present, longwave $7.5-13 \mu \mathrm{m}$ IR thermograpic systems with the uncooled microbolometric arrays (FPA) are most popular in the industry and the science. A measurement of the effective emissivity specially prepared steel samples with modern IR system has been made. Figure 1 shows thermal images of the steel test samples.
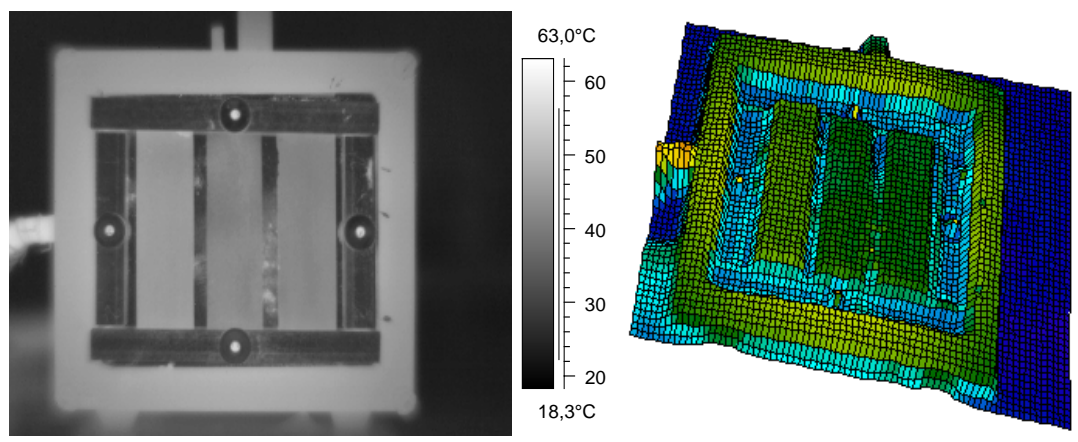

Fig. 1. Thermal imagings of the steel test sample (sand blasted surfaces) 


\section{2. Surface topography}

Surface profilometry is for many years a well-known method of topography inspection. Based on multiprofile representation [8] or spiral sampling [9] gives a three-dimensional image of the surface. It is also possible to evaluate topography parameters and it is a proven fact $[10,11,12]$ that they represent surface properties much better. Among surface parameters there are the ones representing vertical, horizontal and hybrid properties, as well as functions describing surface behaviour [13]. Basing on knowledge concerning emission a number of topography parameters were chosen for possible relation with emissive properties. First parameters from material ratio curve were used. Among them we chose indexes representing volumes (material and void) as potentially good representation of emissive properties. Second a developed area and its relation with sampling area were chosen. Third there was na idea to use isotropy. Finally, as shown earlier from references, a ratio of mean spacing of asperities calculating on the level of reference element and Sz. This was named $\mathrm{Er}$ as emission properties coming from roughness. As in our case all the tested specimen were flat this element was a plane. Values of parameters were calculated after polynominal filtering. Emissivity coming from measurements are shown as $\varepsilon_{\text {eff. }}$

\section{Results and discussion}

For periodic surface which in our case was a milled surface two very different examples were chosen. One surface was relatively smooth (milling 1) while the other was very rough (milling 2). Values of some topography parameters and emission coefficient for both surfaces were shown in table 1. Photo simulations of both surfaces were shown on figure 4. These images also confirm how different were these two surfaces. Figure 2 shows axonometric view of surface named milling 1 while figure 3 shows surface milling 2. As we can see from table 1 (where Sq - root mean square deviation of the surface; St - maximum peak height; Ssk - skewness of topography height distribution; Sku - kurtosis of topography height distribution; Sz 10 point surface height), emissivity does not change much with surface roughness in amplitude meaning. Though difference in asperities is about ten times we can see practically no difference in emissivity. Most probably this is due to the fact that regardless height, asperities have very similar summit angle. Also all the parameters connected with material ratio and developed area do not show any correlation with emissivity.

Tab. 1 Parameters of milled surfaces

\begin{tabular}{|c|c|c|}
\hline Parameter & Milling 1 & Milling 2 \\
\hline Sq & 3,76 & 14,71 \\
\hline St & 32,3 & 80,4 \\
\hline Ssk & 0,003 & 0,421 \\
\hline Sku & 3,76 & 2,23 \\
\hline Sz & 27,7 & 71,9 \\
\hline Er & 1,87 & 3,067 \\
\hline$\varepsilon_{\text {eff }}$ & 0,10 & 0,11 \\
\hline Er / $\varepsilon_{\text {eff }}$ & 18,70 & 27,88 \\
\hline isotropy & 9,9 & 10,2 \\
\hline
\end{tabular}




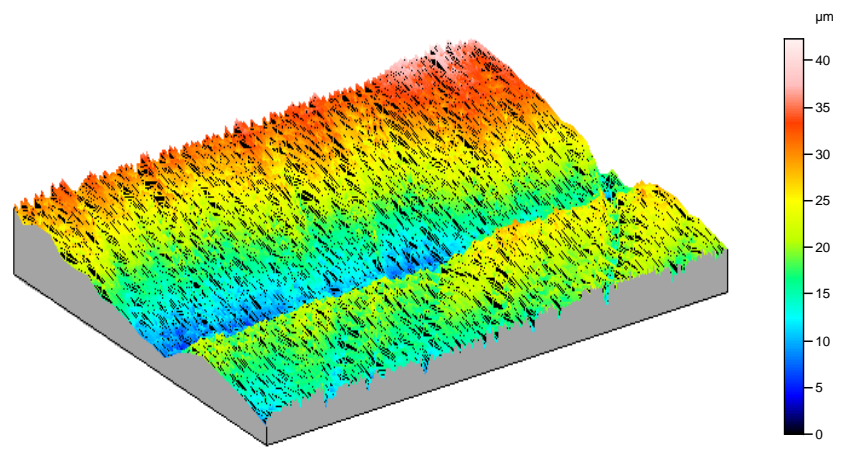

Fig. 2. Axonometric view of surface named milling 1

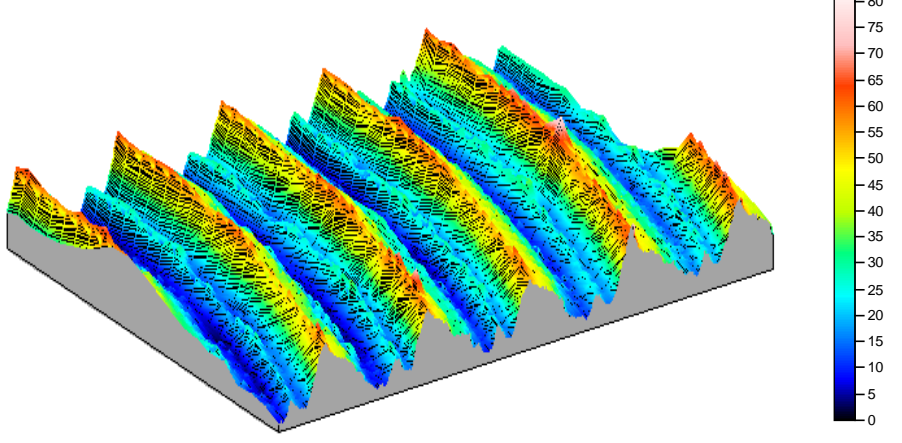

Fig. 3. Axonometric view of surface named milling 2

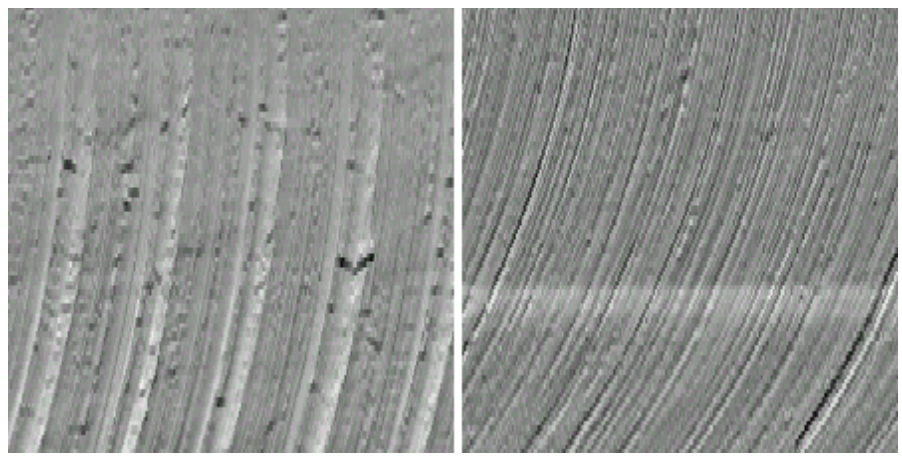

Fig. 3. Photo simulation of milled surfaces. 
The only parameter that can reflect these properties in this case is isotropy. Both surfaces show very similar isotropy or anisotropy rather. There is than a dominant direction in which and this can be connected with heat disspersion from the surface. Isotropy graph for such a surface was shown on figure 5 .

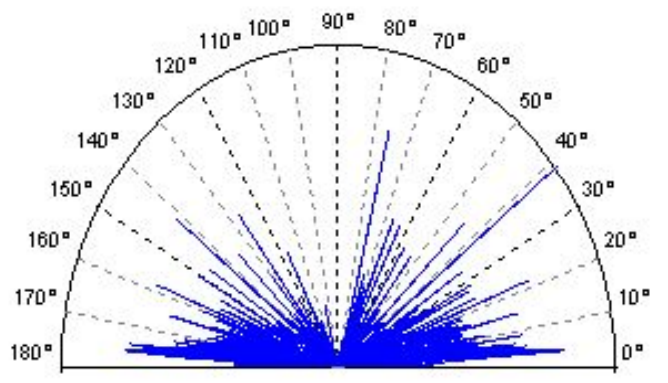

Fig. 5. Isotropy graph for surface milling 2.

A totally different situation can be observed at isotropic surface e.g. a sand blasted one. Parameter values for three typical sand blasted surfaces were presented in table 2.

Tab. 2. Parameters of sand blasted surfaces.

\begin{tabular}{|c|c|c|c|}
\hline Parameter & Sand blasting 1 & Sand blasting 2 & Sand blasting 3 \\
\hline Sq & 5,02 & 7,36 & 3,64 \\
\hline St & 62,0 & 74,8 & 49,9 \\
\hline Ssk & $-0,529$ & $-0,602$ & $-1,022$ \\
\hline Sku & 4,06 & 4,49 & 6,84 \\
\hline Sz & 49,3 & 63,9 & 45,3 \\
\hline Er & 1,36 & 1,23 & 1,17 \\
\hline$\varepsilon_{\text {eff }}$ & 0,46 & 0,40 & 0,38 \\
\hline Er / $\varepsilon_{\text {eff }}$ & 2,959 & 3,075 & 3,079 \\
\hline isotropy & 61,6 & 52,9 & 63,9 \\
\hline
\end{tabular}

Figure 6 shows axonometric view of one of our sand blasted surfaces while figure 7 shows photo images of all of them.

Here from table 2, emission properties also does not change much with surface roughness in amplitude meaning. Sometimes even amplitude parameters grow when emissivity drop down. Also in this case parameters connected with material ratio and developed area do not show any correlation with emissiveity. This surfaces are different from milled ones and isotropy cannot be used to describe heat emission from the surface. However, emission coming from roughnes i.e. ratio of mean spacing to Sz show very good correlation with emissivity. As it was calculated in table one ratio of $\mathrm{Er}$ to $\varepsilon_{\text {eff }}$ which is a ratio of emissive properties calculated and measured for all surfaces remain at the same level with very small differences. A question emerges why isotropy is not suitable for surfaces of that kind. In our opinion this is due to the fact that shape of asperities is very different from milled surface what effects in a different heat transfer and emission. Asperities are much sharper and holes caused by sand direct IR rays very randomly, what can be seen from isotropy graph (fig. 8). 


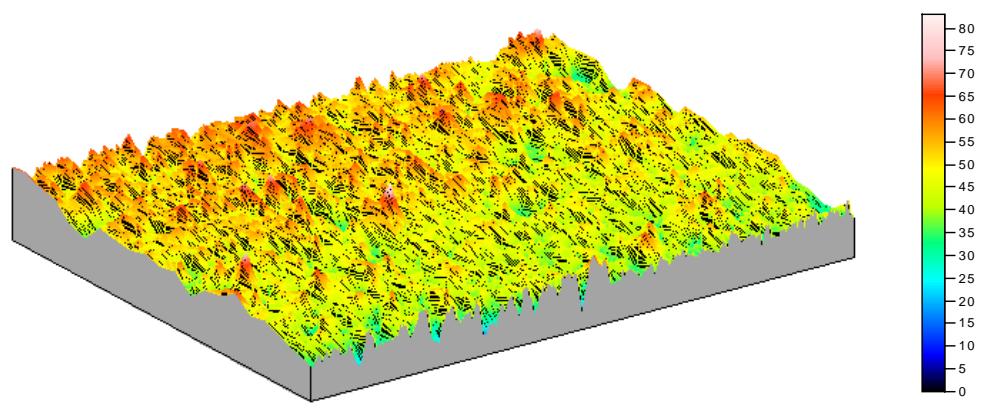

Fig. 6. Axonometric view of sand blasted surface 2.
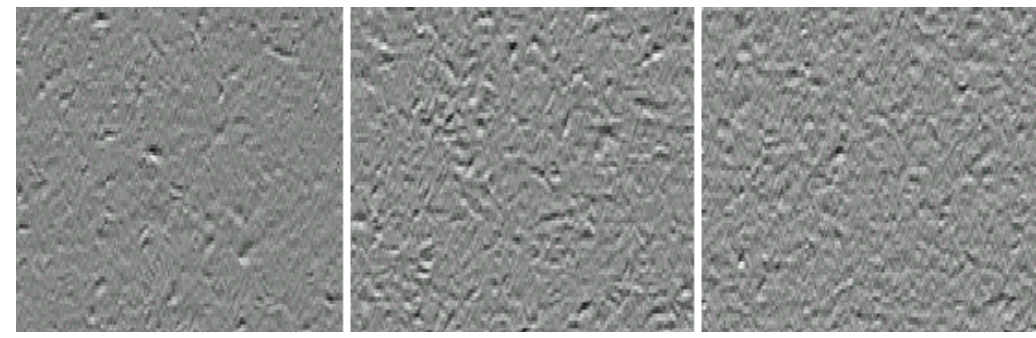

Fig. 7. Photo simulation of sand blasted surfaces.
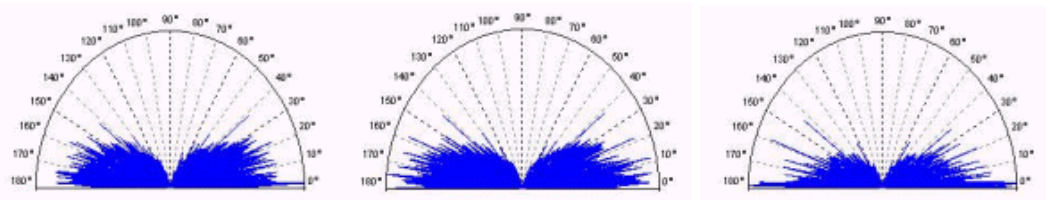

Fig. 8. Isotropy graphs for sand blasted surfaces.

The analysis show that generally it is very difficult to predict emissivity of surface basing on its roughness. It is surely related to many other aspects. Still it is possible to make a relatively good assessment knowing its isotropic properties and calculating ratios of parameters. This can be very useful in diagnosis of machine tools, where a lot of surfaces were milled and - especially with older machines - castings have structure similar to the one that we obtained after sand blasting. From our research it was found out that for surfaces after different types of machining it is worth to use different methods of emissive properties evaluation. 


\section{Conclusions}

Surface topography is one of the features influencing on emissive properties. For periodic surfaces isotropy seems to be a good measure of theoretical emissive properties. Here even big changes in amplitude parameters do not significantly change emissive properties. On the other hand for random surfaces a ratio of mean spacing and Sz seem to be a much better measure of emissive properties. As all the asperities are random and cause random direction of heat rays, isotropy does not give a good image of emission.

\section{References}

[1] A. Otsuka, K. Hosono, R. Taaka, K. Kitagawa, and N. Arai. A survey of hemispherical total emissivity of the refractorymetals in practical use, Energy, $\mathrm{N}^{\circ} 30$, 2005, pp. 535-543.

[2] X. Maldague. Theory and Practice of Infrared Technology for Nondestructive Testing, John Willey \& Sons, Inc., ISBN 0-471-18190-0, 2001.

[3] K. Chrzanowski. Non-contact thermometry-Measurement errors , SPIE PL, Research and Development Treaties, Warsaw, 2000.

[4] S. Poloszyk, and L. Rozanski. Thermographic Diagnosis Station of Machines, 7th International DAAAM Symposium. Vienna 1996, pp. 351-352.

[5] K. Chrzanowski. Problem of Determination of Effective Emissivity of some Materials in MIR Region, Infrared Phys. Technol. Vol 30, N 3, 1995, pp. 679-684.

[6] A. Sala. Radiacyjna wymiana ciepla, WN-T, Warsaw, ISBN 83-204-0367-7, 1982. [7] W. Sabaga and R. Todtenhaupt. The Effect of Roughness on the Emissivity of Precious Metals Ag, Au, Pd, Pt, Rh and Ir, High Temperatures - High Pressure, Vol. 33. $\mathrm{N}^{0} 3,2001$,pp. 261-269.

[8] R.C. Sayles and Thomas. Mapping a small area of a surface, Journal of Physics E: Scientific Instruments, 9, 1976, pp. 855-861.

[9] M. Wiczoroeski. Spiral sampling as a fast way of Data Acquisition in Surface Topography Measurements, International Journal of Machine Tools \& Manufacture, 41, 2001, pp. 2017-2022.

[10] T. Tsukada and T. Kanada. Evaluation of two- and three- dimensional surface roughness profiles and their confidence, Wear, 109, 1986, pp. 69-78.

[11] T. Klimczak. Origins, magnitude and statistical significance of differences between roughness parameters of two- and three-dimensional characteristics, Wear, 156, 1992, pp. 19-31.

[12] M. Wieczorowski, A. Cellary and J.Chajda. Parameters for three dimensional surface texture character analysis, Proceedings of the Third International Symposium on Measurement Technology and Intelligent Instruments ISMTII'96. Hayama, Japan 1996, pp. 209-216. 